\title{
Reconstruction of Mandible with the Use of Free Flaps after Segmental Resection with Exarticulation
}

\author{
Lilia Pavlovna Yakovleva', Mikhail Alekseevich Kropotov' ${ }^{2}$, Ludmila \\ Grigorjevna Zhukova ${ }^{3}$, Vladimir Anatolievich Sobolevskiy ${ }^{4}$, Artem \\ Valerevich Khodos ${ }^{5 *}$ and Petr Aleksandrovich Gavrischuk ${ }^{5}$ \\ ${ }^{1}$ Head of Head and Neck Surgery Department, Moscow Clinical Scientific Center n.a \\ A.Loginov, Moscow, Russia \\ ${ }^{2}$ Head of Head and Neck Surgery Department, National Medical Research Center of \\ Oncology Named After N.N. Blokhin, Moscow, Russia \\ ${ }^{3}$ Deputy Director of Oncology, Moscow Clinical Scientific Center n.a A.Loginov, \\ Moscow, Russia \\ ${ }^{4}$ Head of Reconstruction and Plastic Department, National Medical Research Cen- \\ ter of Oncology Named After N.N. Blokhin, Moscow, Russia \\ ${ }^{5}$ Department of Head and Neck Surgery, Moscow Clinical Scientific Center n.a \\ A.Loginov, Moscow, Russia \\ *Corresponding Author: Artem Valerevich Khodos, Department of Head and Neck \\ Surgery, Moscow Clinical Scientific Center n.a A.Loginov, Moscow, Russia.
}

Received: September 15, 2021

Published: December 10, 2021

(C) All rights are reserved by Artem

Valerevich Khodos., et al.

\begin{abstract}
Introduction: Segmental resection of the mandible is often used in patients with advanced malignant tumors of oral cavity and primary tumors of the lower jaw. Violation of mandibular arch after the surgical treatment leads to the problems with chewing, swallowing, speech and significant esthetic defects and deformation of lower face area. This circumstances force the surgeon to choose a safe method of reconstruction that can replace large bone and soft-tissue defects and guarantee an adequate time of postoperative treatment.
\end{abstract}

Materials and Methods: A total of 23 patients with primary and secondary tumors of mandible were included into our study. All had undergone segmental resection of the lower jaw with exarticulation with one-time reconstruction with free bone flaps over the period of 14 years from 2003 to 2018.

Results: The described in the article method of joint reconstruction guarantees reliable fixation of the new-formed articular head and recreate temporomandibular joint.

Conclusion: The use of bone transplants can restore the anatomy of maxillofacial area, the function of temporomandibular joint and the form of the lower zone of the face after segmental resection of the lower jaw with exarticulation in patients with primary and secondary, benign and malignant tumors.

Keywords: Reconstruction of Mandible; Free Flaps; Segmental Resection; Exarticulation

\section{Introduction}

Segmental resection of the mandible is often used in patients with advanced malignant tumors of oral cavity and primary tumors of the lower jaw [1]. Violation of mandibular arch after the surgical treatment leads to the problems with chewing, swallowing, speech and significant esthetic defects and deformation of lower face area [2]. Even if the oncological treatment is successful, the quality of life is reduced greatly. In cases when surgical treatment is 
performed after radiation, the wound healing is usually complicated due to decrease of regenerative abilities of tissues and contact with the bacterial flora of oral cavity. This circumstances force the surgeon to choose a safe method of reconstruction that can replace large bone and soft-tissue defects and guarantee an adequate time of postoperative treatment.

Reconstruction mandible and soft-tissues at the same time with tumor removal reaches good results and reduces the rehabilitation time. A variety of ways of reconstruction makes a choice of a method complicated. It depends on the size, localization of the defect and the oncological prognosis [2,3]. In patients with advanced tumors, both primary tumors of mandible (that is more often) and oral cancer with bone invasion up to the base of articular process, segmental resection of the lower jaw with exarticulation must be performed.

Reconstruction of temporomandibular joint is an actual problem of modern of maxillofacial surgery because this joint plays a significant role in chewing and phonation [4]. Both own tissues and synthetic materials are used nowadays.

There are many publications devoted to the arthroplasty of temporomandibular joint and mandible after resection with exarticulation [4-6]. Most authors positively estimate this method and focus on its long term benefits [7]. At the same time, some authors describe a lot of late complications such as fracture of the endoprosthesis fixing part, migration of its head to the middle cranial fossa, eruption of reconstruction plates [8,9]. Preservation of articular meniscus, joint capsule and chewing muscles is an important point in reconstruction of temporomandibular joint and can be performed safe from the oncological point of view in most cases. It improves the rehabilitation and reduces the number of complications. Besides, it is necessary to cover the prosthesis and the reconstructive plate well with the surrounding soft tissues, fix the head of the prosthesis with the articular capsule and follow the principles of rigid fixation of the whole complex to the remaining mandible fragment [10].

The modern approach includes a combination of revascularized bone flaps (mostly fibula free flap) and one-pole titanium prosthesis of the articular head with the use of computer modeling and manufacturing (CAD/CAM). It allows reconstructing the lower jaw defect and restoring the function of temporomandibular joint [11]. But the number of complications is up to $10.6 \%$ due to the use of allogenic material [12]. The significant cost of this method is another problem and makes it inappropriate in some cases. An alternative way is the use of autogenic bone transplants with articular head modeling. Many authors emphasize on the idea that there is no need in absolute congruence in the joint because its function defines its anatomy. This way of reconstruction has no complications typical for the use of allogenic materials. The comparison of different methods of modeling the articular head showed no significant differences [13-19]. Thus, the problem of choosing the best way of reconstruction after segmental resection of mandible with exarticulation after surgical treatment of oral cancer and primary tumors of lower jaw has not been solved yet and a lot of its aspects is still discussed in scientific society.

\section{Materials and Methods}

A total of 23 patients with primary and secondary tumors of mandible were included into our study. All had undergone segmental resection of the lower jaw with exarticulation with one-time reconstruction with free bone flaps over the period of 14 years from 2003 to 2018. The need for exarticulation were determined by the tumor localization in the ramus of mandible and in the corpus of mandible together with the ramus (Picture 1 and 2). The invasion into the mandibular canal in patients with squamous cell carcinoma of oral cavity lead to the need in hemimandibulectomy according to the oncological principles. At the same time, the tumor lesion of the articular head was noted only twice $(8.7 \%)$.

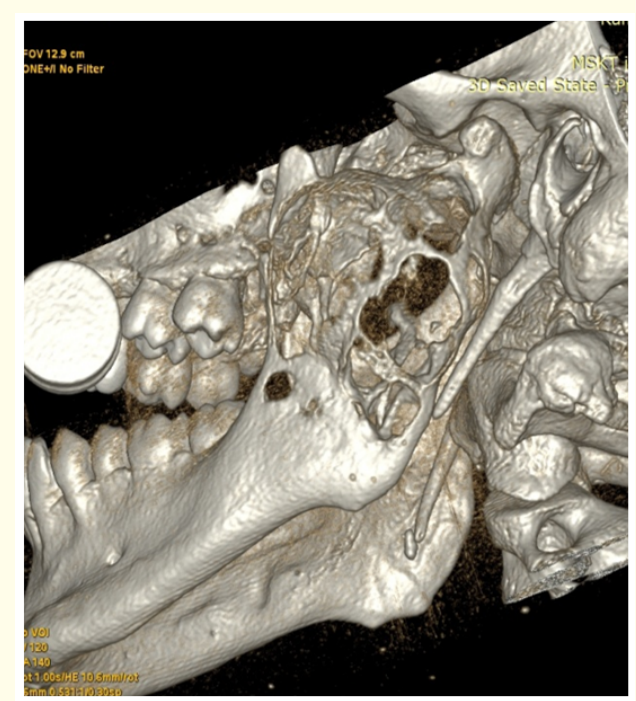

Picture 1: CT scan. Ameloblastoma of the mandibular ramus. 


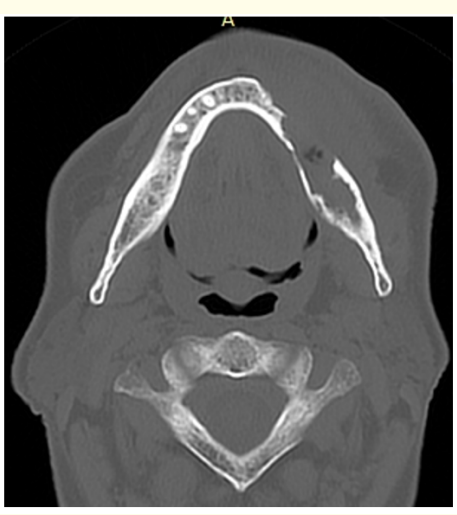

Picture 2: CT scan. Squamous cell carcinoma of alveolar process. The bone destruction of the mandibular body and ramus.

15 males and 8 females aged from 15 to 65 years with a medium age of 37.3 were included into our study. The patients with primary tumors of mandible determined the relatively young medium age.

Segmental resection of lower jaw with exarticulation was mostly performed in patients with primary tumors of mandible: 20 cases (87\%), moreover, non-epithelial malignant tumors were met: 15 cases (65\%), distributed mainly between osteosarcomas in 9 cases and chondrosarcomas in 4 cases. Much less often parosteal sarcoma ( 1 case) and neurosarcoma (1 case) were met. 5 benign tumors were diagnosed: 3 ameloblastomas and 2 giant cell tumors.

Secondary lesion of mandible in patients with squamous cell carcinoma with spread over the spongious bone up to the mandibular canal and ramus was diagnosed in 3 cases.

Significant predominance of primary tumors of mandible in our study is determined by the particularities of bone invasion and spread of epithelial and non-epithelial tumors (Picture 3 and 4). Squamous cell carcinoma spreads along the cortical plate with subsequent local bone invasion and only after that involves the spongious bone. Primary tumors of the lower jaw initially affect the spongious bone, it determines the lager scope of surgical intervention.

The majority of patients (11 cases), considering the above-described clinical features and treatment approach, did not receive prior treatment. In two cases of osteosarcoma neoadjuvant chemo-
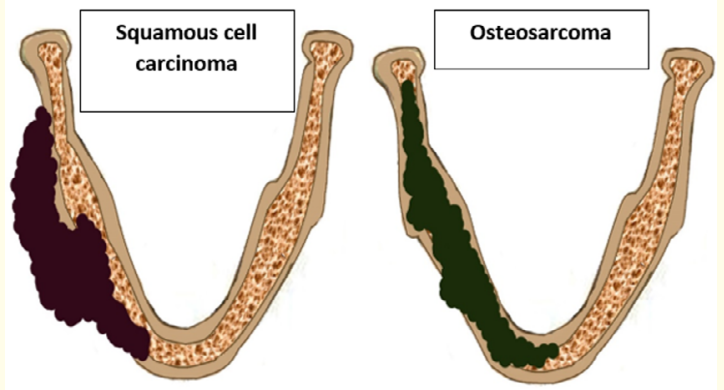

Picture 3 and 4: Particularities of bone invasion and spread of epithelial (3) and non-epithelial tumors (4).

therapy was performed. Once surgical treatment was performed in case of revisional osteosarcoma after previous operation. In one observation a rare radioinduced osteosarcoma of mandible developed 8 years after radiotherapy for tongue cancer. In patients with squamous cell carcinoma of oral cavity, surgery was performed as the first step of complex treatment and went along with lymph node dissection. According to our data isolated lesion of the mandibular ramus was diagnosed five times (21.7\%), mainly in patients with benign tumors and only once in a case of parosteal sarcoma. In the majority of patients was found the lesion of mandibular body and ramus (13 patients, $56 \%$ ). And in 5 patients $(21.7 \%)$ tumors involved mental part and the opposite side of the mandibular body.

Segmental resection of the mandible with exarticulation was performed according to the generally excepted method with resection of mucosa and soft tissues depending on the tumor spread. While the resection special attention was paid to the preservation of the joint capsule, meniscus and, if possible, masticator muscles. This structures are then used for the reconstruction of the lower jaw and the joint. For the comfortable and accurate adaptation of the transplant, we used interosseous fixation.

The method of modeling the transplant and its fixation is an important problem. In our work we never used stereolithographic scull models giving precedence to easier and cheaper method of forming the transplant according to the size and form of the resected fragment of the mandible (Picture 13). Interosseous fixation in complete occlusal contact and pre-made aluminum template are required in this situation. In cases when reconstructive titanium plate was used, the shape of the transplant and the number of oste- 
otomies was determined directly while adopting the transplant to the plate. Moreover, the adequate blood supply and stable fixation is more important than the congruence of the osteotomy planes.

We used two types of bone transplants in our work: iliac and fibula ones:

1. Iliac free flap was used in 5 patients with isolated defects of the mandibular ramus. Distal margin of the flap was formed according to the shape of the resected jaw with the use of an oscillating saw and a bur and that moved to the glenoid fossa. Joint capsule and masticatory muscles were sutured to the transplant's muscle clutch and periosteum. After that proximal margin of the transplant was fixed with two titanium mini plates to the remaining fragment of the lower jaw. Then microanastomoses were performed between the vessels of the transplant and the vessels on the neck (most often with facial artery and vein) (Picture 5-12).

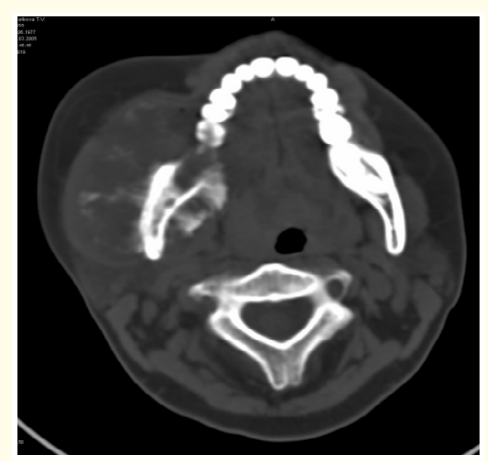

Picture 5: CT scan of the mandible. Giant cell tumor with lower jaw and articular process lesion. Large soft-tissue component.

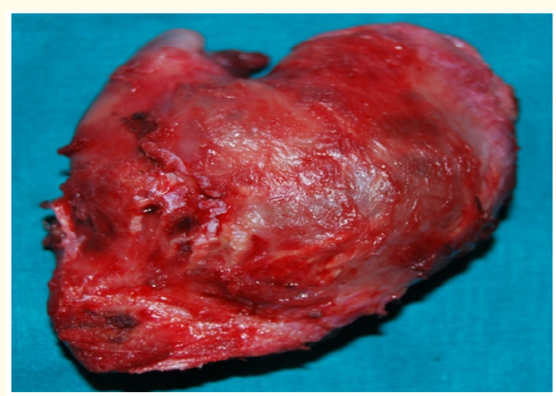

Picture 6: Gross specimen. Resected mandibular ramus together with the articular process and tumor. Deformation of the articular head.

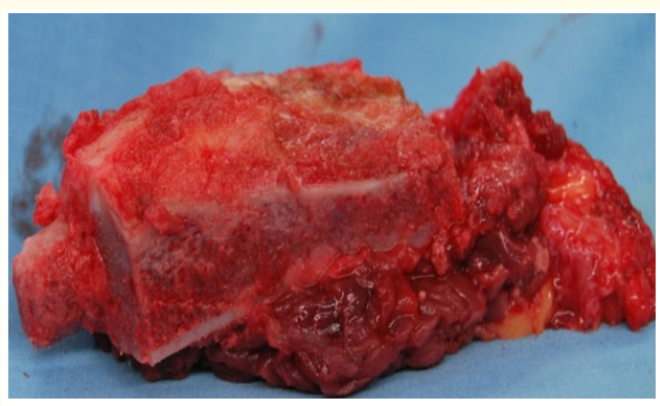

Picture 7: Iliac free flap with muscle clutch, formed according to the size of the defect. The formed articular head marked by arrow.

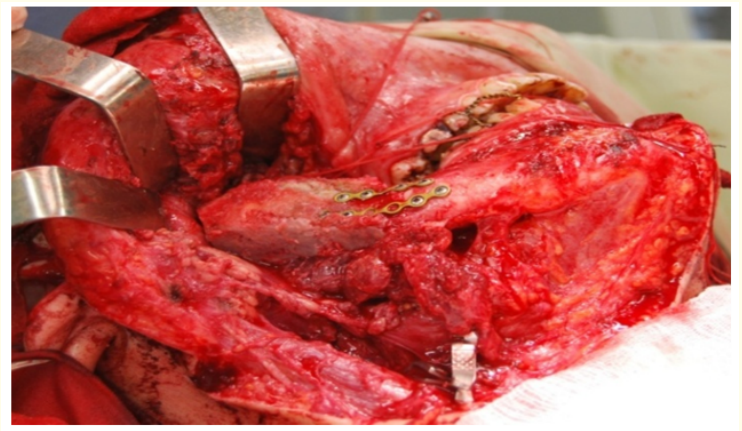

Picture 8: Adopted iliac free flap. The articular head placed in the glenoid fossa and fixed by suturing the joint capsule, masticatory muscles and the transplant's muscle clutch. The bone part is fixed to the remaining fragment of the lower jaw with mini plates.

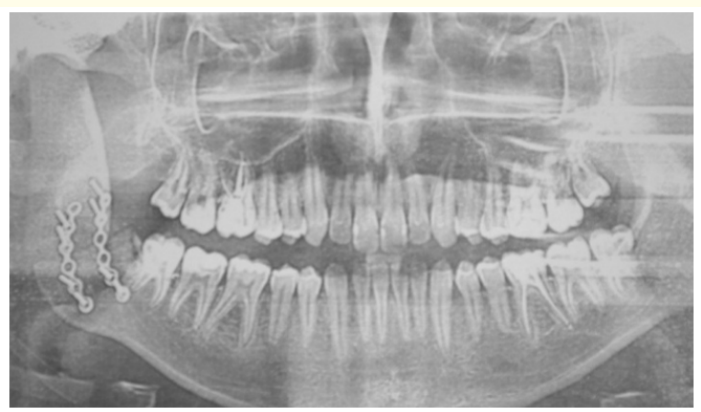

Picture 9: Orthopantomogram. 


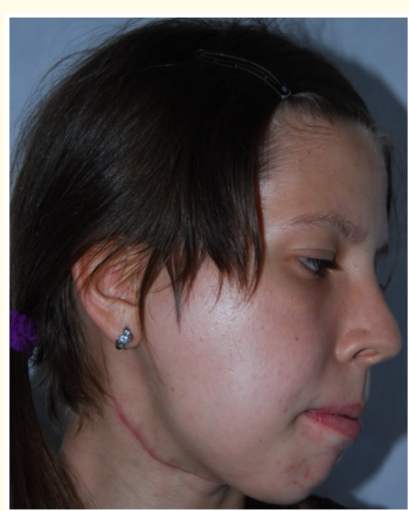

Picture 10: Patients facial appearance.

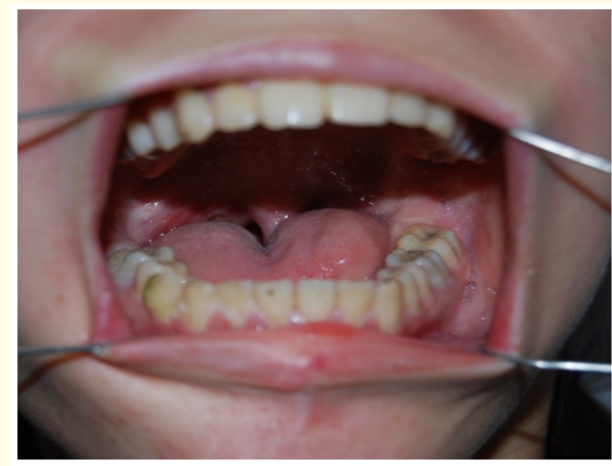

Picture 11: Same patient. Mouth opening.

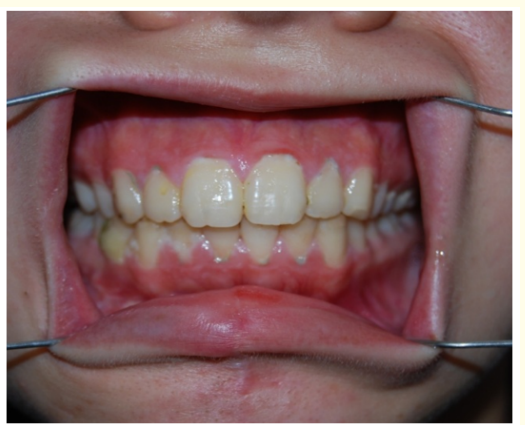

Picture 12: Interdigitation.

2. Fibula free flap was used in 18 cases for the defects of the mandibular ramus and body or ramus, body, mental part and body from the opposite side. Thus, this flap is the method of choice in patients with extended defects. Besides, in makes possible to reconstruct not only the bone defect, but the mucosa too. The number of osteotomies varied from 1 to 4, depending on the form and size of the defect. The bone fragments between each other and with the remaining fragment of the lower jaw were fixed with the use of mini plates or with the pre-formed reconstructive plate. The distal margin was also formed with the oscillating saw, moved to the glenoid fossa and fixed according to the method described above (Picture 13-23). The microanastomoses were made at the same side or at the opposite one, depending on the size of the transplant.

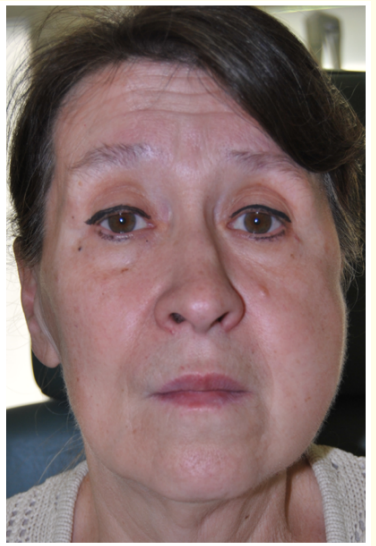

Picture 13: Patient with osteogenic sarcoma of the power jaw. Deformation of the left cheek and parotideomasseteric region.

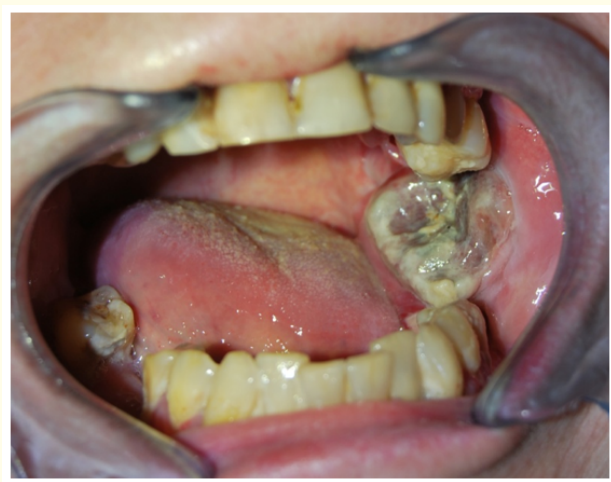

Picture 14: Oral cavity. Tumor-related deformation of the alveolar process. Retromolar area and cheek lesion. 


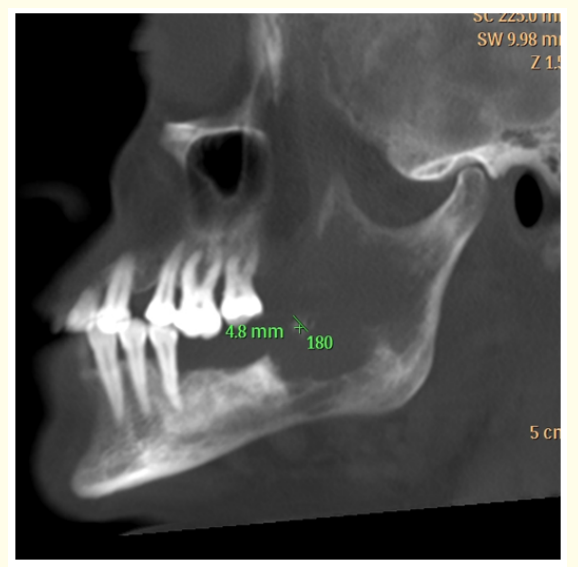

Picture 15: CT scan, reconstruction. Bone destruction of the mandibular body and ramus. Destruction of the cortical plate.

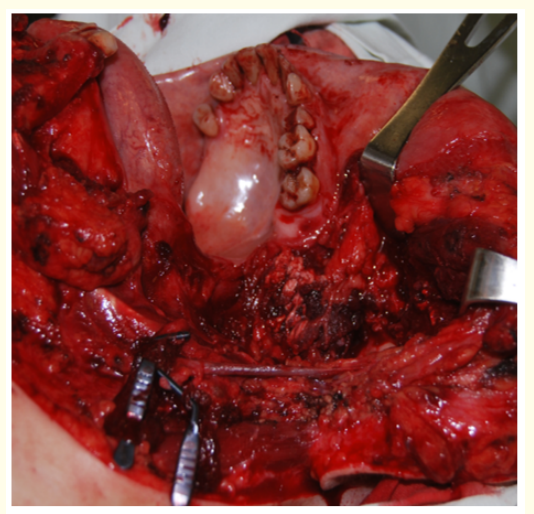

Picture 16: Surgical wound after segmental resection of mandible with exarticulation and resection of surrounding soft tissues.

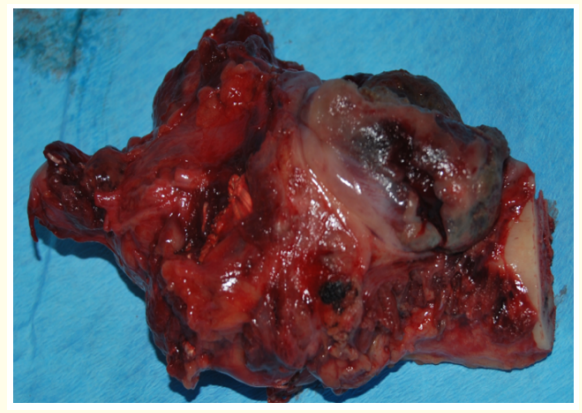

Picture 17: Gross specimen. Lower jaw fragment with articular head and surrounding soft tissues.

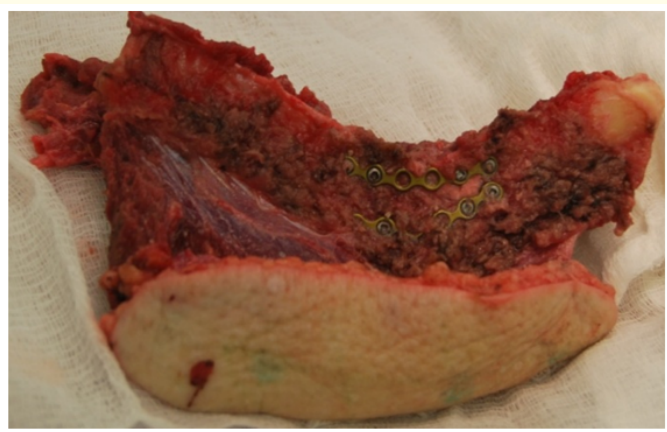

Picture 18: Formed according to the size and shape of the lower jaw combined fibula free flap. The articular head formed at the transplants distal margin marked by arrow.

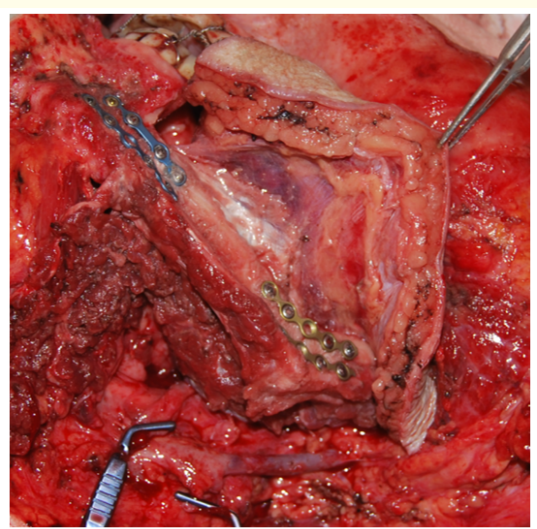

Picture 19: Fibular free flap replacing the defect of the lower jaw. The distal margin placed in the glenoid fossa, the proximal one fixed with mini plates to the lower jaw.

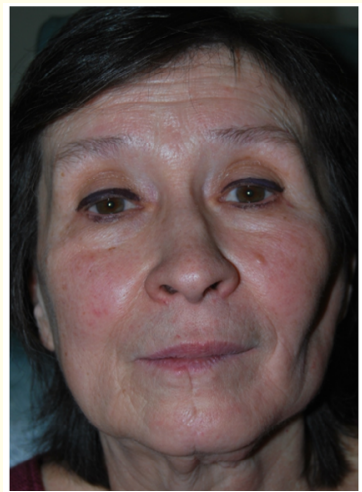

Picture 20: Patients facial appearance after 18 months and 6 courses of adjuvant chemotherapy. 


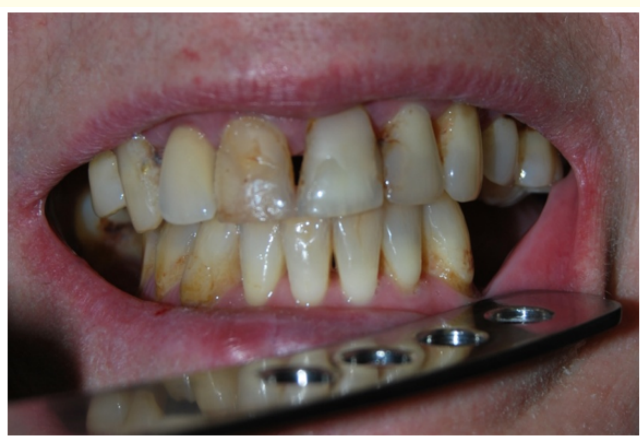

Picture 21: Oral cavity. Interdigitation.

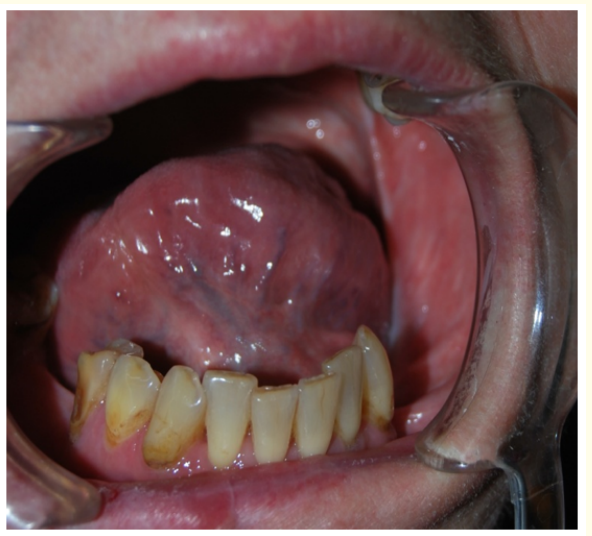

Picture 22: Oral cavity. Sufficient moth opening.

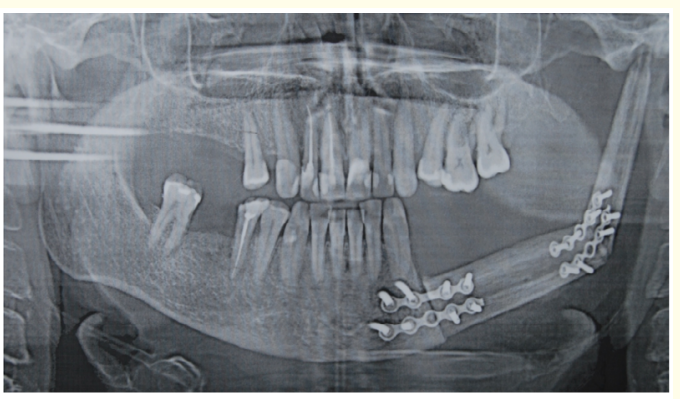

Picture 23: Orthopantomogram. The lower jaw defect replaced by the fibular free flap. The newly formed articular head of the transplant is congruent with the articular fossa.

\section{Results and Discussion}

As to the results of our study, the described method of joint reconstruction between the articular fossa, meniscus with the surrounding tissues and distal part of the transplant guarantees reliable fixation of the new-formed articular head and recreate temporomandibular joint. Proper restoration of the anatomy with preservation of muscle structures helps to restore the articular function. Important to say that in all of the described cases, regardless of the type of transplant, full opening of the mouth noted, both in early and late postoperative period. Great importance in the rehabilitation plays the possibility of early activation of the lower jaw function. Besides, the preservation of the contour and form of the lower face zone allows achieving good esthetical results. Only in 2 patients we had to perform corrective plastic surgery: in one case due to significant facial asymmetry we performed resection of the transplant's angle for the right shape of the lower jaw and another time contour plastic of the parotideomasseteric zone with allogenic graft.

Complications noted in 4 cases (17.4\%). Inadequate blood flow in the skin part of the fibula flap at the end of the operation was noted once. The skin was resected and the defect of mucosa replaced by nasolabial flap. Total necrosis of the fibula flap was noted once. It was removed and replaced by musculocutaneous flap. Paresis of the orbital branch of the facial nerve noted in two cases.

\begin{tabular}{|l|c|c|}
\hline \multirow{2}{*}{ The size of the lower jaw lesion } & \multicolumn{2}{|c|}{ Type of reconstruction } \\
\cline { 2 - 3 } & Iliac free flap & $\begin{array}{c}\text { Fibula free } \\
\text { flap }\end{array}$ \\
\hline Ramus & 5 & -- \\
\hline Ramus+body & -- & 13 \\
\hline Ramus+body+mental part+body & -- & 5 \\
\hline Total & 5 & 18 \\
\hline
\end{tabular}

Table 1: Types of reconstruction of the mandible in aspect of the size of lower jaw lesion.

The analysis of oncological results in this study is a difficult task due to the significant difference in the tumor spread and morphology. Never the less, no disease recurrence noted in patients with benign tumors.

In patients with squamous cell carcinoma with lower jaw lesion recurrence of the disease noted in 1 case of 3 , it caused death. In the group of non-epithelial malignant tumors in 7 cases $(46.6 \%)$ of disease recurrence noted: 2 lung metastases (13.3\%), 3 primary tumor recurrences $(20.0 \%)$ and 2 primary tumor recurrences together with lung metastases (13.3\%). In 2 cases local recurrence was treated surgically, but never the less lung metastases were 
diagnosed later. The highest rate of recurrences was found in patients with osteosarcoma: 5 of 9 cases (55.6\%).

\section{Conclusion}

The use of bone transplants can restore the anatomy of maxillofacial area, the function of temporomandibular joint and the form of the lower zone of the face after segmental resection of the lower jaw with exarticulation in patients with primary and secondary, benign and malignant tumors.

\section{Bibliography}

1. Ralph W Gilbert. "Reconstruction of the oral cavity; past, present and future”. Oral Oncology 108 (2020): 104683.

2. Kiran Kakarala., et al. "Mandibular reconstruction". Oral Oncology 77 (2018): 111-117.

3. Andreas F Mavrogenis., et al. "Microsurgical reconstruction of complex oromandibular defects: An update". Injury 50.5 (2019): S117-S122.

4. Mario J Imola and Aaron Liddell. "Temporomandibular joint reconstruction". Current Opinion in Otolaryngology and Head and Neck Surgery 24.4 (2016): 336-342.

5. Ardalan Ebrahimi and Bruce G Ashford. "Advances in temporomandibular joint reconstruction". Current Opinion in Otolaryngology and Head and Neck Surgery 18.4 (2010): 255-260.

6. M K Wax., et al. "A retrospective analysis of temporomandibular joint reconstruction with free fibula microvascular flap". Laryngoscope 110.6 (2000): 977-981.

7. E Daniel and J Browne. "Minimizing complications in the use of titanium condylar head reconstruction prostheses". Otolaryngology-Head and Neck Surgery 130.3 (2004): 344-350.

8. A Patel and R Maisel. "Condylar prostheses in head and neck cancer reconstruction". Archives of Otorhinolaryngology-Head and Neck Surgery 127.7 (2001): 842-846.

9. Majeed Rana Reconstruction of mandibular defects - clinical retrospective research over a 10-year period". Head and Neck Oncology 3 (2011): 23.

10. ER Carlson. "Disarticulation resections of the mandible: a prospective review of 16 cases". Journal of Oral and Maxillofacial Surgery 60.202 (2002): 176-181.

11. L Ciocca., et al. "A CAD $\backslash$ CAM prototyped temporomandibular condole connected to a bony plate to support a free fibula flap in patients undergoing mandiblectomy: a pilot study with 5 years of follow up". Journal of Cranio-Maxillofacial Surgery 44.7 (2016): 811-819.
12. R Marx., et al. "Outcome analysis of mandibular condylar replacements in tumor and trauma reconstruction: a prospective analysis of 131 cases with long-term follow-up". Journal of Cranio-Maxillofacial Surgery 66 (2008): 2515-2523.

13. Ivana Petrovic., et al. "Long-term functional and esthetic outcomes after fibula free flap reconstruction of the mandible". Head Neck 41.7 (2019): 2123-2132.

14. J Weitz., et al. "Accuracy of mandibular reconstruction by three-dimensional guided vascularised fibular free flap after segmental mandibulectomy". British Journal of Oral and Maxillofacial Surgery 54 (2016): 506-510.

15. Wei FC., et al. "Fibular osteoseptocutaneous flap: anatomic study and clinical application". Plastic and Reconstructive Surgery 78 (1986): 191.

16. Yoshimura H., et al. "Infra zygomatico-coronoid fixation in a segmental mandibular reconstruction with a free vascularised flap: a simple and correct repositioning method without interfering with reconstructive and microsurgical procedures". Head Neck 38.11 (2016): 1679-1687.

17. Ritschl LM., et al. "Functional outcome of CAD \CAM assisted versus conventional microvascular fibular free flap reconstruction of the mandible: a retrospective study of 30 cases". Journal of Reconstructive Microsurgery 18 (2017): 1055-1060.

18. Sawh-Martinez R., et al. "Improved temporomandibular joint position after 3-dimensional planned mandibular reconstruction". Journal of Oral and Maxillofacial Surgery 75.1 (2017): 197-206.

19. Tarsitano A., et al. "Short term outcomes of mandibular reconstruction in oncological patients using a CAD \CAM prosthesis including a condyle supporting a fibular free flap". Journal of Cranio-Maxillofacial Surgery 45.2 (2017): 330-337.

\section{Assets from publication with us}

- Prompt Acknowledgement after receiving the article

- Thorough Double blinded peer review

- Rapid Publication

- Issue of Publication Certificate

- High visibility of your Published work

Website: www.actascientific.com/

Submit Article: www.actascientific.com/submission.php

Email us: editor@actascientific.com

Contact us: +919182824667 